\title{
The role of clockwork orange in the circadian clock of the cricket Gryllus bimaculatus
}

\author{
Yasuaki Tomiyama', Tsugumichi Shinohara', Mirai Matsuka', Tetsuya Bando², Taro Mito ${ }^{3}$ and Kenji Tomioka ${ }^{1 *}$ (D)
}

\begin{abstract}
The circadian clock generates rhythms of approximately $24 \mathrm{~h}$ through periodic expression of the clock genes. In insects, the major clock genes period (per) and timeless (tim) are rhythmically expressed upon their transactivation by CLOCKVCYCLE, with peak levels in the early night. In Drosophila, clockwork orange (cwo) is known to inhibit the transcription of per and tim during the daytime to enhance the amplitude of the rhythm, but its function in other insects is largely unknown. In this study, we investigated the role of cwo in the clock mechanism of the cricket Gryllus bimaculatus. The results of quantitative RT-PCR showed that under a light/dark (LD) cycle, cwo is rhythmically expressed in the optic lobe (lamina-medulla complex) and peaks during the night. When cwo was knocked down via RNA interference (RNAi), some crickets lost their locomotor rhythm, while others maintained a rhythm but exhibited a longer free-running period under constant darkness (DD). In $\mathrm{CWO}^{\mathrm{RNAi}}$ crickets, all clock genes except for cryptochrome 2 (cry2) showed arrhythmic expression under DD; under LD, some of the clock genes showed higher mRNA levels, and tim showed rhythmic expression with a delayed phase. Based on these results, we propose that cwo plays an important role in the cricket circadian clock.
\end{abstract}

Keywords: Circadian clock, Clockwork orange, Clock gene, Cricket, cry2, Molecular oscillation, Locomotor rhythm

\section{Introduction}

Most insects exhibit daily rhythms in their physiology, including in their locomotor activity. The rhythms are driven by an endogenous oscillatory mechanism called the circadian clock, which generates approximately 24-h rhythms that persist in the absence of environmental cues [1]. The clock is based on the rhythmic expression of clock genes such as per, tim, Clock $(C l k)$, and cycle (cyc). It is generally thought that the CLOCK (CLK)/CYCLE (CYC) heterodimer activates the transcription of per and tim by binding to the Ebox located upstream of their promoter region [1, 2]. The protein products of per and tim accumulate

\footnotetext{
* Correspondence: tomioka@cc.okayama-u.ac.jp

${ }^{1}$ Graduate School of Natural Science and Technology, Okayama University, Okayama 700-8530, Japan

Full list of author information is available at the end of the article
}

during the night, form PER/TIM heterodimers and enter the nucleus to inhibit the transcriptional activity of CLK/CYC late at night. In the fruit fly Drosophila melanogaster, the transcriptional activator CLK is also rhythmically expressed by a mechanism that includes vrille (vri) and Par domain protein $1 \varepsilon(P d p 1 \varepsilon)$ [3, 4]. Both vri and Pdp1E are transactivated by CLK/CYC through the E-box in the late day to early night, similar to per and tim. Soon after, the transcribed vri mRNA is translated to its product protein VRI, which suppresses the transcription of $C l k$ during the night, while $P d p 1 \varepsilon$ is translated later and activates $C l k$ transcription in the late night to early day [3-5]. This mechanism leads to the rhythmic expression of CLK with peak levels during the day.

The per/tim oscillatory loop is known to receive fine tuning by clockwork orange (cwo) in Drosophila, which is

(C) The Author(s). 2020 Open Access This article is licensed under a Creative Commons Attribution 4.0 International License, which permits use, sharing, adaptation, distribution and reproduction in any medium or format, as long as you give appropriate credit to the original author(s) and the source, provide a link to the Creative Commons licence, and indicate if changes were made. The images or other third party material in this article are included in the article's Creative Commons licence, unless indicated otherwise in a credit line to the material. If material is not included in the article's Creative Commons licence and your intended use is not permitted by statutory regulation or exceeds the permitted use, you will need to obtain permission directly from the copyright holder. To view a copy of this licence, visit http://creativecommons.org/licenses/by/4.0/. The Creative Commons Public Domain Dedication waiver (http://creativecommons.org/publicdomain/zero/1.0/) applies to the data made available in this article, unless otherwise stated in a credit line to the data. 
a clock gene that forms a feedback loop independent of the per/tim loop [6-8]. cwo is rhythmically expressed under the regulation of CLK/CYC with a peak during the night, and its protein product $\mathrm{CWO}$ is thought to bind the E-box as a competitor of CLK/CYC, inhibiting the expression of E-box regulated genes, including per, tim, and cwo itself [9]. For per and tim, CWO function terminates their transcription late at night and suppresses their transcription during the day, causing the production of a higher oscillation amplitude of transcription. A similar function has also been hypothesized for the mammalian homologs, the Differentiated embryo chondrocyte (Dec) genes $[10,11]$. Drosophila CWO has also been suggested to activate the transcription of per, tim, vri, and $P d p 1 \varepsilon$, which are target genes of CLK/CYC, and to be involved in posttranslational control of clock proteins [12]. However, the detailed mechanisms for these functions of $c w o$ have yet to be explored.

Although cwo has been found in some other insects, including the monarch butterfly Danaus plexippus, the fire ant Solenopsis invicta, and the jewel wasp Nasonia vitripennis [13-15], little is known about its function in insect species other than Drosophila. To better understand the role of $c w o$, comparative studies using different phylogenetic classes of insects are required, as there are considerable differences in the oscillatory mechanism of the clock among insects $[1,2]$. Here, we investigated the role of cwo in the cricket Gryllus bimaculatus. The oscillatory mechanism of this hemimetabolous insect differs from that of Drosophila in several aspects. Instead of $C l k, c y c$ is rhythmically expressed, while $C l k$ is rhythmically expressed when $c y c$ is downregulated [16]. In addition to the per/tim loop, the mechanism includes cry genes that form another feedback loop, which can oscillate independently of the per/tim loop [17]. In this study, we first detected the presence of the cwo gene in G. bimaculatus and then examined its role in both behavioral rhythms and molecular oscillatory mechanisms using RNA interference (RNAi). We found that cwo plays an essential role in the molecular oscillatory mechanism of $G$. bimaculatus but that there is a compensatory mechanism that can retain behavioral rhythmicity even when the function of cwo is disrupted.

\section{Materials and methods}

\section{Experimental animals}

All experiments were performed with adult male crickets (Gryllus bimaculatus) that were reared in the laboratory or purchased. The crickets were kept under controlled conditions of $12 \mathrm{~h}$ light and $12 \mathrm{~h}$ darkness (LD 12:12, light: 0600-1800, Japan Standard Time) at a constant temperature of $25 \pm 1.0^{\circ} \mathrm{C}$.

\section{cDNA cloning}

We first searched for cwo, E75, and HR3 genes in our RNA-seq data. The sequence data reported for other insect species were used for these searches. The cDNA fragments of the identified genes were obtained via RT-PCR as follows. Total RNA was extracted with TRIzol ${ }^{\circ}$ Reagent (Ambion, Austin, TX, USA) from 10 adult optic lobes consisting of lamina and medulla neuropiles collected at ZT 6 (ZT stands for zeitgeber time and ZT0 corresponds to lights-on and ZT12 to lights-off). Total RNA ( $4.5 \mu \mathrm{g})$ was used for reverse transcription to obtain cDNA using the PrimeScript ${ }^{\bullet}$ RT reagent Kit (Takara, Otsu, Japan). Using single-stranded cDNA as a template, we performed PCR with EmeraldAmp PCR Master Mix (Takara) and the primers listed in Table 1 . The PCR conditions employed were 40 cycles of $30 \mathrm{~s}$ at $95^{\circ} \mathrm{C}$ for denaturation, $30 \mathrm{~s}$ at $55^{\circ} \mathrm{C}$ for annealing, and $1 \mathrm{~min} 30 \mathrm{~s}$ at $72^{\circ} \mathrm{C}$ for extension. The amplified sequences were analyzed by BLAST (https://blast.ncbi.nlm.nih.gov/Blast.cgi).

\section{RNA measurement}

qPCR and RT-PCR were used to measure mRNA levels. Total RNA was extracted and purified from 2 to 6 optic lobes of adult male crickets with TRIzol Reagent (Invitrogen, Carlsbad, CA, USA). To remove genomic DNA contamination, the total RNA was treated with DNase I (Invitrogen). Approximately $500 \mathrm{ng}$ of total RNA from each sample was reverse transcribed using random hexamers and PrimeScript RT reagent Kit (Takara). Realtime PCR was performed with the Mx3000P Real-Time PCR System (Stratagene, La Jolla, CA, USA) using FastStart Universal SYBR Green Master (Roche, Tokyo, Japan), including SYBR Green and primers designed for cwo, per (GenBank/EMBL/DDBJ Accession No. BAG48878), tim (BAJ16356), Clk (AB738083), cyc (AB762416), vri (LC512907), Pdp1 (LC512908), E75 (LC536674), HR3 (LC536673), and rpl18a (DC448653) (Table 1). In all cases, a single expected amplicon was confirmed via melting analysis. The quantification was performed based on a standard curve obtained with a known amount of template. The results were analyzed using the software associated with the instrument. The values were then normalized with those of rpl18a at each time point. The results of 3-6 independent experiments were used to calculate the mean \pm SEM.

\section{RNAi}

Double-stranded RNA (dsRNA) for the cricket clock genes cwo, per, tim, cry2, Clk, and cyc and for the control gene DsRed2 derived from a coral species (Discosoma sp.), were synthesized using a MEGAscript High Yield Transcription kit (Ambion, Austin, TX, USA). For the clock genes, cDNAs prepared as described above were used as templates for PCR, which was performed using ExTaq DNA 
Table 1 PCR primers used for quantitative RT-PCR and dsRNA synthesis. The primers tagged with T7 or T3 promoter sequences were used for PCR amplification for dsRNA synthesis. T7 and T3 sequences are underlined

\begin{tabular}{|c|c|c|}
\hline Primers & Forward & Reverse \\
\hline \multicolumn{3}{|l|}{ For qPCR } \\
\hline per & 5'-AAGCAAGCAAGCATCCTCAT-3' & 5'-CTGAGAAAGGAGGCCACAAG-3' \\
\hline tim & 5'-GATTATGAAGTCTGTGATGATTGG-3' & 5'-AGCATTGGAGAGAACTGAAGAGGT-3' \\
\hline cry2 & 5'-AGCACCATCACACACTTCACA-3' & 5'-ACACTCAGCGCAATCCACAC-3' \\
\hline Clk & 5'-AATGACCGTAGTCGAGAAAGTGAAG-3' & 5'-TTGCGATGATTGAGGTTGTTG-3' \\
\hline cyc & 5'-GGCCGAAGCTCATAAAGTGG-3' & 5'-AACCGCACAAAGGAACCATC-3' \\
\hline vri & 5'-TCAGCGTGGAGCAAGTGATG-3' & 5'-GGGTACAGCAGCGAGTGTTG-3' \\
\hline Pdp1 & 5'-TCCCGACGACAAGAAGGAG-3' & 5'-AGCGTCTTGTCCCAGAGGTTG-3' \\
\hline E75 & 5'-CACACGCAAGTGGAGGACA-3' & 5'-TTTGTGCGGCTTGTAGGC-3' \\
\hline HR3 & 5'-CATGTTGTACCCATCAAAGGTG-3' & 5'-TGTGGAGAGCTGGAAACTCC-3' \\
\hline$r p / 18 a$ & 5'-GCTCCGGATTACATCGTTGC-3' & 5'-GCCAAATGCCGAAGTTCTTG-3' \\
\hline \multicolumn{3}{|c|}{ For dsRNA synthesis } \\
\hline per & 5'-TAATACGACTCACTATAGGGATGTGGCTTGGAAGATCATT-3' & 5'-AATTAACCCTCACTAAAGGGTCTCCTTAAGCAAATTCTCA-3' \\
\hline $\operatorname{tim}$ & 5'-AATTAACCCTCACTAAAGGGGTAAAGAAGATAGAGAGTAT-3' & 5'-AATTAACCCTCACTAAAGGGTTGGAGAGAACTGAAGAGGT-3' \\
\hline cry2 & 5'-TAATACGACTCACTATAGGGAAGCACACTGTGCATTGGTT-3' & 5'-AATTAACCCTCACTAAAGGGCCGTTCTTTTCGATGATGCT-3' \\
\hline Clk & 5'-TAATACGACTCACTATAGGGTCATAATGAGTTGAGTTCT-3' & 5'-TAATACGACTCACTATAGGGAAGGGGTGTCTGTAATCTT-3' \\
\hline$c y c$ & 5'-TAATACGACTCACTATAGGGCGTGCACTCGTACACTGAGG-3' & 5'-AATTAACCCTCACTAAAGGGAGGTTCTGCTGCTTCTTTCG-3' \\
\hline DsRed2 & 5'-TAATACGACTCACTATAGGGTCATCACCGAGTTCATGCG-3' & 5'-TAATACGACTCACTATAGGGCTACAGGAACAGGTGGTGGC-3' \\
\hline
\end{tabular}

polymerase (Takara). The T7- or T3-containing primers that were used are listed in Table 1 . The amplified fragments of cwo (128 bp), per (456 bp), tim (519bp), cry2 (422 bp), Clk (407 bp), and cyc (450 bp) were extracted with phenol/chloroform, precipitated with ethanol, and then resuspended in Ultra Pure Water (Invitrogen). For DsRed2 dsRNA, the linearized DsRed2 fragment was amplified from pDsRed2-N1 (Clontech, Mountain View, CA, USA) using the primers shown in Table 1 . Using each of these linearized fragments as a template, RNA was synthesized with T7 or T3 RNA polymerase. Synthesized RNAs were extracted with phenol/chloroform, precipitated with isopropanol, and suspended in $50 \mu \mathrm{l}$ of TE solution. The yield and quality of the RNA were assessed by measuring the absorbance with a spectrophotometer (GeneQuant Pro, Amersham Bioscience, Piscataway, NJ, USA), and equal amounts of sense and antisense RNA were mixed. The RNAs were denatured for $5 \mathrm{~min}$ at $100{ }^{\circ} \mathrm{C}$ and were gradually cooled to room temperature for annealing. After ethanol precipitation, the dsRNA obtained was suspended in Ultra Pure Water (Invitrogen) with the final concentration adjusted to $20 \mu \mathrm{M}$. The dsRNA solution was stored at $-80^{\circ} \mathrm{C}$ until use. The dsRNA solution $(760 \mathrm{nl})$ was injected into the abdomen of adult crickets anesthetized with $\mathrm{CO}_{2}$ using a nanoliter injector (WPI, Sarasota, FL, USA).

\section{Locomotor activity recording}

The locomotor activities of the crickets were recorded using the method described previously [18]. In brief, the adult crickets were individually housed in a transparent plastic box $(18 \times 9 \times 4.5 \mathrm{~cm})$ containing a rocking substratum. The movement of crickets resulted in the movement of the substratum, which was recorded every $6 \mathrm{~min}$ by a computerized system. Food and water were provided ad libitum. The actographs were placed in an incubator in which light was provided via a cool white fluorescent lamp connected to an electric timer. The raw data were displayed in conventional double-plotted actograms to judge activity patterns and were statistically analyzed using the chisquare periodogram [19] in Actogram J (freely available at http://actogramj.neurofly.de/) [20]. If a peak of the periodogram appeared above the 0.05 confidence level, with the power value (height of the peak above the confidence level) greater than or equal to 10 and the width of the peak greater than or equal to 2, then the period of the peak was designated as statistically significant [21].

\section{Statistics}

The differences in mean mRNA levels between different time points were compared using one-way analysis of variance (ANOVA) followed by a post hoc Tukey's test. We also used CircWave (ver. 1.4) (available at http:// www.rug.nl/fwn/onderzoek/programmas/biologie/chronobiologie/downloads/index) to determine the significance of daily and circadian rhythmicity. When the results of both ANOVA and CircWave analysis were 
statistically significant, the rhythm was designated as significant. When the result of only one analysis was significant, the pattern was designated quasi-rhythmic. To compare the means of two groups, a t-test was used. The mRNA levels of crickets treated with dsRNA targeting clock genes were compared at each ZT or CT (CT stands for circadian time and CT0 corresponds to projected lights-on and CT12 to projected lights-off) with a control treated with dsDsRed2 with ANOVA followed by Dunnett's test. The significance level was set at $P<$ 0.05 for all statistics.

\section{Results}

Molecular cloning and structural analysis of cwo

To obtain a cDNA fragment of $c w o$, we searched for a sequence homologous to known sequences of insect $c w o$ genes in our RNA-seq data. We found two fragments, one encoding a 172 aa long protein (GenBank/EMBL/DDBJ Accession No. LC536675), including a bHLH domain, and the other encoding a 123 aa long protein (LC536676), including a Hairy Orange domain (Fig. 1a). We confirmed the sequences by DNA sequencing, followed by RT-PCR, using the primers synthesized for fragment amplification.

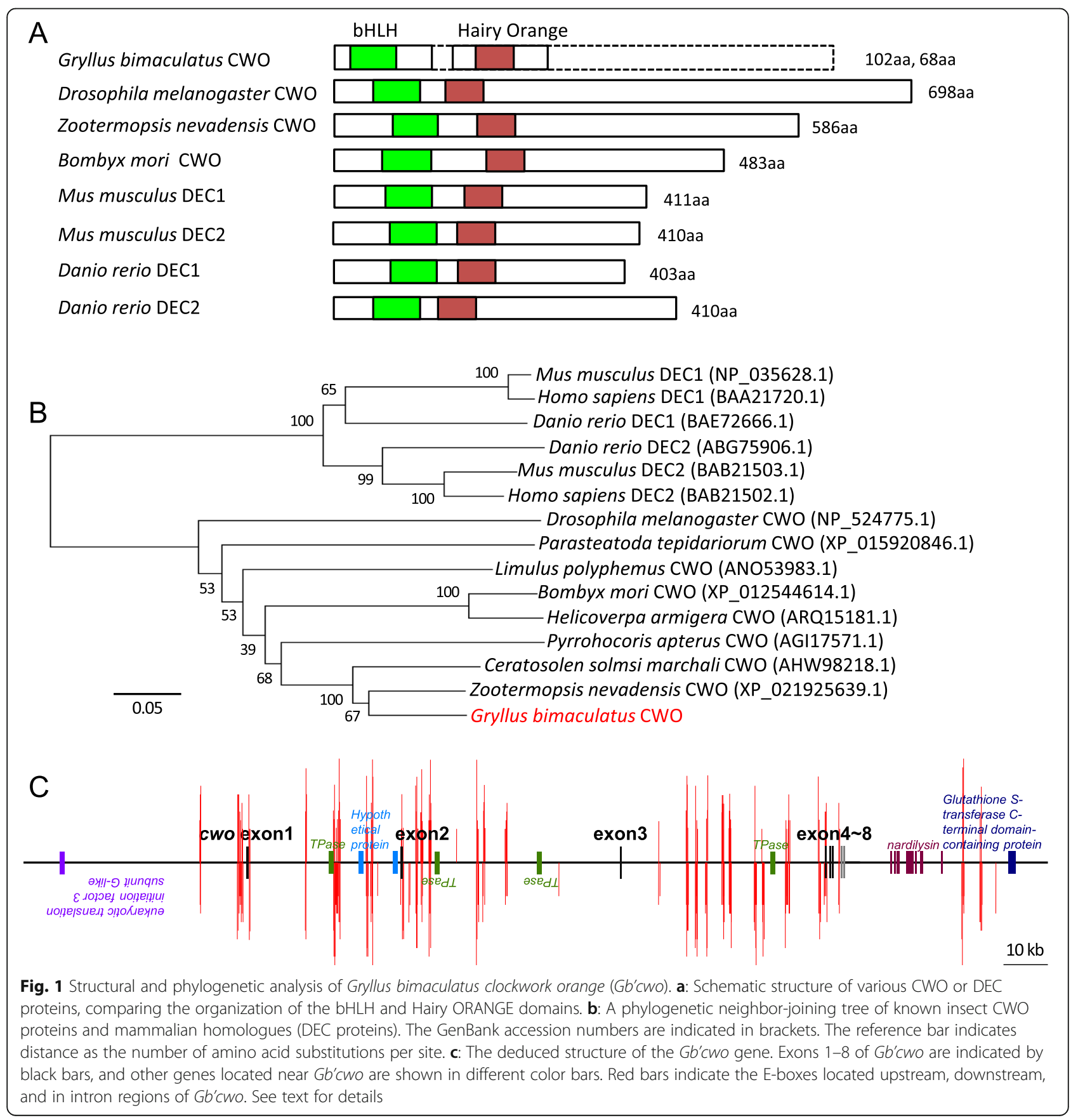


A BLAST database search indicated that the amino acid sequence of the bHLH domain of Gryllus bimaculatus CWO ( $G b^{\prime}{ }^{\prime} \mathrm{CWO}$ ) has $88.7-96.2 \%$ identity and that of the Hairy Orange domain has $34.1-92.5 \%$ identity with the amino acid sequences of known insect CWOs, including those of the termite Zootermopsis nevadensis (XP 021925639.1), moth Bombyx mori (XP_012544614.1), and fruit fly Drosophila melanogaster (NP_524775.1) (Table 2). The bHLH domain also has relatively high identity (43.146.3\%) and similarity (70.4-80.4\%) to the vertebrate homologs of CWO, namely, DEC1 and DEC2, in Danio rerio (BAE72666.1, ABG75906.1), Mus musculus (NP 035628.1, BAB21503.1), and humans (BAA21720.1, BAB21502.1) (Table 2). We thus concluded that the obtained fragments are of Gryllus bimaculatus cwo (Gb'cwo), which belongs to the bHLH-ORANGE family. A phylogenetic tree based on the amino acid sequences of CWO from known insects and those of DEC1 and DEC2 from some vertebrates revealed that $G b^{\prime} \mathrm{CWO}$ forms a clade with CWOs of other insects and is closely related to that of the termite $Z$. nevadensis (Fig. 1b).

We then analyzed the structure of the Gb'cwo gene and nearby cis elements. The structure of the Gb'cwo gene was deduced using draft genome sequence data of G. bimaculatus and the cDNA sequences of known insect $c$ wo genes. Figure 1c shows the expected exon/intron structure of the Gb'cwo gene. Gb'cwo was presumed to consist of 8 exons. We explored cis elements in the $50 \mathrm{~kb}$ regions upstream of exon 1 and downstream of exon 8 using Cister (Cis element cluster finder, https://zlab.bu.edu/ mfrith/cister. shtml) and found many E-boxes in both sense and antisense strands, especially within the $10 \mathrm{~kb}$ region upstream of exon 1.

\section{Tissues expressing cwo}

To determine which tissues express $c w o$, we measured cwo mRNA levels in the optic lobe, protocerebral lobe (brain), subesophageal ganglion, and compound eyes by
qPCR. The samples were collected at midday (ZT6), midnight (ZT18), subjective midday (CT6), and subjective midnight (CT18). As shown in Fig. 2, cwo mRNA was detected in all of these tissues. Under the LD cycle, the expression was highest in the compound eye and lowest in the brain and subesophageal ganglion. The cwo RNA levels in the compound eye, optic lobe, and brain changed daily, with the highest amplitude (3.4-fold) in the compound eye (Fig. 2a). The day-night changes in the optic lobe and brain were 1.8-fold and 2.0-fold, respectively. The subesophageal ganglion also showed higher cwo levels at night, but the difference was not significant.

Under constant darkness (DD), daily expression profiles were basically reproduced in tissues collected at CT6 and CT18 (Fig. 2b). In the compound eye, optic lobe, and brain, the cwo levels were higher at CT18, and the circadian changes were 3.2-fold, 1.4-fold, and 2.1fold, respectively. The expression in the subesophageal ganglion did not show a significant rhythm.

\section{Daily expression of cwo mRNA}

We first examined the expression profile of $c w o$ mRNA in the cricket clock tissue, the optic lobe, under LD 12: 12. The qPCR results showed that cwo mRNA was rhythmically expressed (Fig. 3a, Table 3). It was expressed at a low level during the daytime, with the expression gradually increasing around light-off and peaking in the middle of the night. The profile was similar to those of per and tim $[18,22]$ (see also Figs. 5 and 6). A similar expression pattern was observed 2 days after the crickets were transferred to DD (Fig. 3b, Table 3). The mRNA levels were similar between LD and DD conditions.

We then examined the effects of RNAi of other clock genes, including per, tim, cry2, Clk, and cyc, on the mRNA levels of $c w o$. As a control, we tested the effects of RNAi of DsRed2. The cwo expression profiles in the $\operatorname{Ds} \operatorname{Red} 2^{\mathrm{RNAi}}$-treated crickets were similar to those of the

Table 2 Identity (\%) and similarity (\%) of bHLH domain and Hairy ORANGE domain of Gb'CWO with other insect CWOs and vertebrate DECs

\begin{tabular}{lllll}
\hline Species & \multicolumn{2}{l}{ bHLH domain } & & \multicolumn{2}{l}{ Hairy ORANGE domain } \\
\cline { 2 - 4 } & Identitiy (\%) & Similarity (\%) & & Identity (\%) \\
\hline Zootermopsis nevadensis CWO & 96.2 & 100 & 92.5 & 97.5 \\
Bombyx mori CWO & 92.5 & 98.1 & 57.5 & 77.5 \\
Drosophila melanogaster CWO & 88.7 & 96.2 & 34.1 & 48.8 \\
Danio rerio DEC1 & 46.3 & 70.4 & 26.2 & 40.5 \\
Danio rerio DEC2 & 46.3 & 70.4 & 26.8 & 43.9 \\
Mus musculus DEC1 & 43.1 & 80.4 & 31.4 & 68.6 \\
Mus musculus DEC2 & 43.1 & 80.4 & 29.7 & 78.4 \\
Homo sapiens DEC1 & 43.1 & 80.4 & 31.4 & 68.6 \\
Homo sapiens DEC2 & 43.1 & 80.4 & 37.8 & 75.7 \\
\hline
\end{tabular}



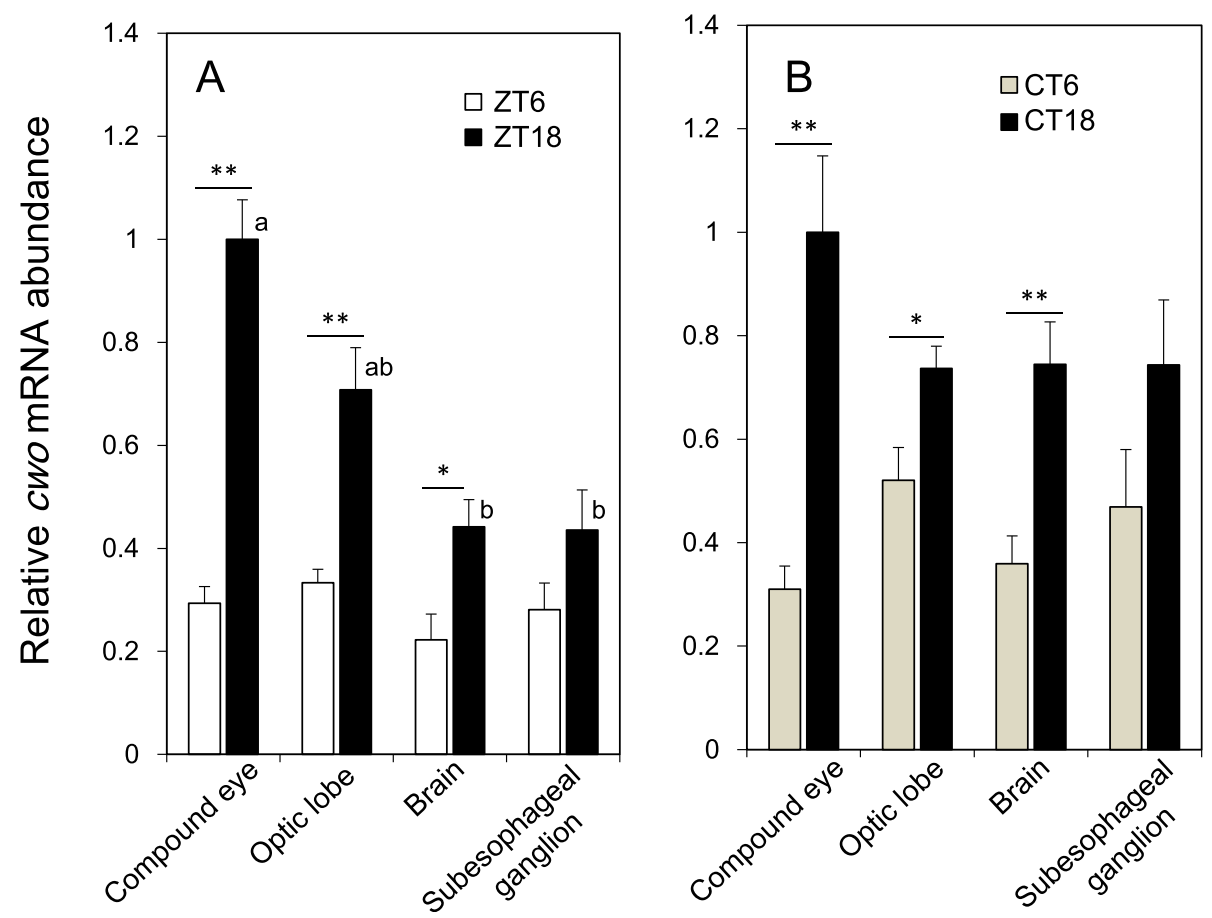

Fig. 2 cwo expression in the compound eye, optic lobe, brain, and subesophageal ganglion of the cricket Gryllus bimaculatus under LD (a) and DD (b). The mRNA expression levels showed a significant day-night change in the compound eye, optic lobe, and brain, with the levels being significantly higher at night (ZT18) or subjective night (CT18) $\left(^{*} P<0.05,{ }^{* *} P<0.01\right.$, t-test). The mRNA level and amplitude were greatest in the compound eye under both LD and DD conditions. The values are shown as relative to the mRNA levels of $r p / 18 a$ and are normalized to the highest value measured in the compound eye. Different letters indicate a significant difference detected by Tukey's test $(P<0.05)$. See text for details

untreated crickets under both LD and DD (Fig. 3a, b), and no significant difference was observed at all ZTs and most CTs (t-test, $P>0.05$ ); the exceptions were CT2 and CT22, in which the values were lower than those of untreated crickets ( $\mathrm{t}$-test, $P<0.05$ ). RNAi of per, tim, and $c y c$ resulted in the loss of daily rhythm of cwo expression, and $p r^{\mathrm{RNAi}}$ and $c y c^{\mathrm{RNAi}}$ downregulated the mRNA levels of cwo compared to DsRed2 ${ }^{\mathrm{RNAi}}$, while $C l k^{\mathrm{RNAi}}$ resulted in a quasi-rhythmic expression of $c w o$ with a peak in the early day (Fig. 3c, Table 3). The results suggest that cwo is under the regulation of the circadian clock. Interestingly, however, dscry 2 treatment showed almost no effect on the rhythmic expression of cwo (Fig. 3c, Table 3).

\section{Effects of cwo dsRNA treatment on locomotor rhythm}

To investigate the role of cwo in the cricket clock, we first examined the effects of systemic $c w o^{\mathrm{RNAi}}$ on the mRNA levels of cwo in the optic lobe under LD and DD conditions. Under both conditions, $c w o^{\mathrm{RNAi}}$ treatment significantly reduced the cwo mRNA levels to below or near the basal level in controls treated with $D s R e d 2^{\mathrm{RNAi}}$ and eliminated the rhythmic expression of $c w o$ that was evident in control crickets (Fig. 3a, b, Table 3).

We then tested the effects of $c w o^{\text {RNAi }}$ on circadian locomotor rhythms. We injected cwo dsRNA into the abdomen or bilateral compound eyes in 22 and 10 adult male crickets, respectively, and recorded their locomotor activity, first under LD conditions for a week and then under DD. Because the results of these two treatments were similar, we pooled the results. We also recorded the locomotor activity of DsRed2 $2^{\mathrm{RNAi}}$-treated crickets as a control $(n=16)$. As shown in Fig. 4a, most of the control crickets $(n=15)$ showed a clear nocturnal rhythm in LD and a free-running rhythm with a period that was slightly shorter than $24 \mathrm{~h}$ in the ensuing DD, while one cricket became arrhythmic. The average free-running period of the rhythmic crickets was $23.5 \pm 0.3(\mathrm{SD}) \mathrm{h}$. In $c w o^{\mathrm{RNAi}}$ crickets $(n=32), 23$ showed a nocturnal rhythm similar to that of controls (Fig. 4b, d), while 9 showed diurnal activity in LD (Fig. 4c). In DD, the 9 diurnal crickets and one nocturnal cricket became arrhythmic, with activity dispersed over $24 \mathrm{~h}$ (Fig. 4b, c). The remaining 22 crickets showed a free-running rhythm with a period $(24.4 \pm 1.5 \mathrm{~h})$ that was significantly longer than that of $D s R e d 2^{\mathrm{RNAi}}$ controls (Fig. $4 \mathrm{~d}$ ) (t-test, $P<0.05$ ). The proportion of arrhythmic crickets in $c w o^{\mathrm{RNAi}}$ treatments was significantly higher than that in the DsRed2 ${ }^{\mathrm{RNAi}}$ control (chisquare test, $P<0.05$ ).

\section{Effects of cwo dsRNA treatment on the clock molecular machinery}

To investigate the role of $c w o$ in the clock oscillatory machinery, we examined the effects of $c w o^{\mathrm{RNAi}}$ on the 


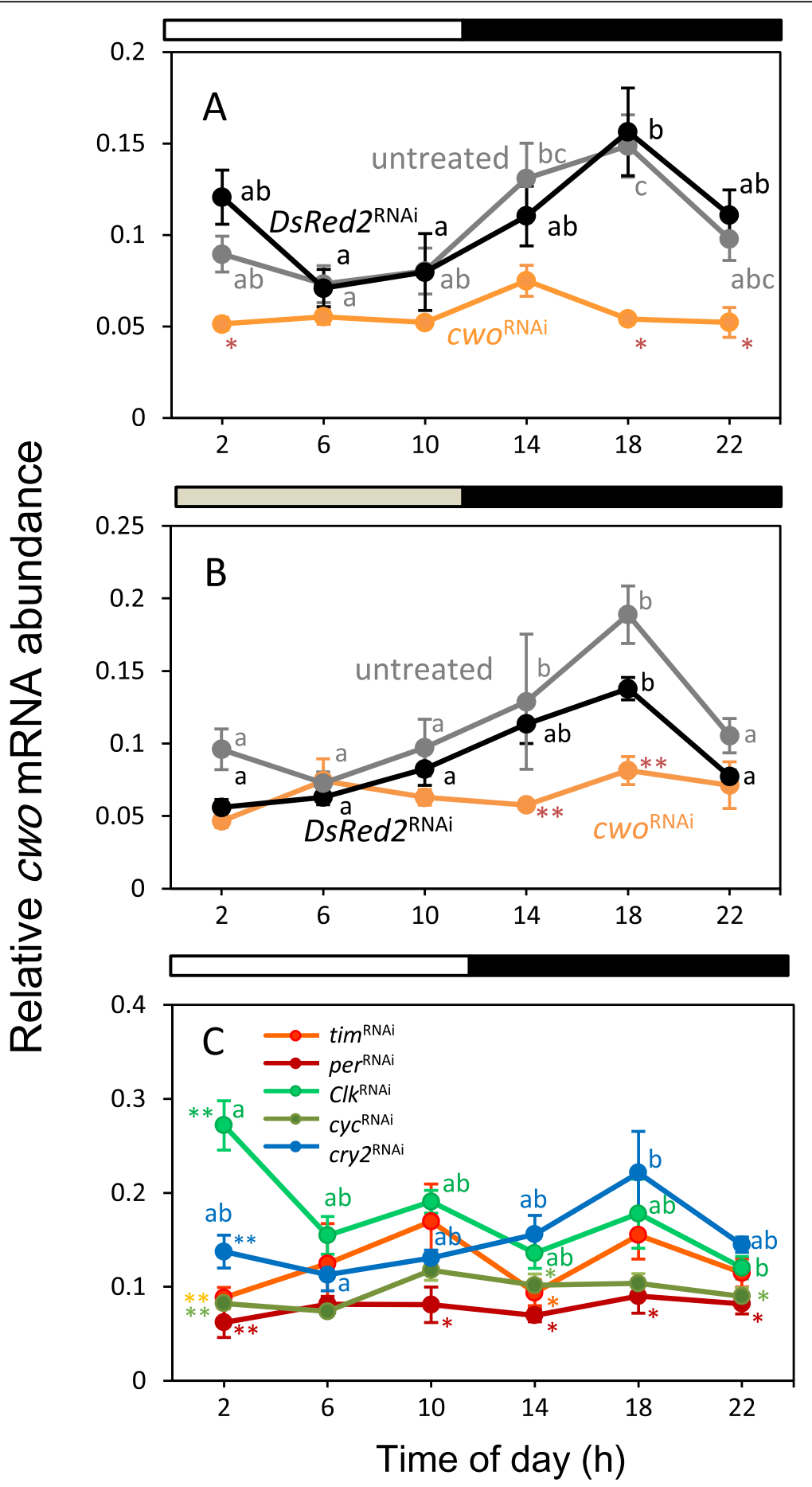

Fig. 3 Expression rhythms of cwo in the optic lobe of the cricket Gryllus bimaculatus and the effects of clock gene RNAi on the expression of cWo. a: cwo is rhythmically expressed in the optic lobe with a peak level at midnight in both untreated (gray) and DsRed2 ${ }^{\text {RNAi }}$-treated crickets (black) under LD. dscwo strongly suppressed cwo mRNA levels and eliminated its rhythmic expression (orange). b: cwo is also rhythmically expressed under DD in the optic lobe of untreated (gray) and DsRed2 ${ }^{\mathrm{RNA}}$-treated crickets (black). dscwo again suppressed cwo mRNA levels and eliminated its rhythmic expression (orange). c: Effects of dsRNA of clock genes, per, tim, Clk, cyc, and cry2 on cwo expression under LD. per ${ }^{\text {RNAi }}$,

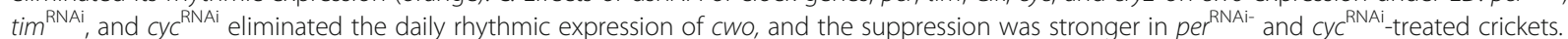
$C I k^{\text {RNAi }}$ and $c r y 2^{\text {RNAi }}$ did not eliminate the rhythm. $c r y 2^{\text {RNAi }}$ treatment had no significant effects on the expression rhythm of $c w 0$, while $C / k^{\text {RNAi }}$ induced its phase shift to peak at ZT2. Asterisks indicate significant differences compared to the control treated with dsDsRed2 ${ }^{*} P<0.05$, ${ }^{* *} P<$ 0.01 , Dunnett's test). Different lowercase letters indicate that the values differ significantly from each other (Tukey's test, $P<0.05$ ). White, gray, and black bars above the panel indicate light (white), subjective day (gray), and dark/subjective night (black) fractions, respectively. See text for details 
Table 3 Results of statistical analyses of cwo expression in untreated crickets and those treated with RNAi of DsRed2 or clock genes under light-dark cycle (LD) or constant darkness (DD)

\begin{tabular}{|c|c|c|c|c|}
\hline \multirow[t]{2}{*}{ Treatment } & \multicolumn{3}{|c|}{ ANOVA } & \multirow{2}{*}{$\begin{array}{l}\text { CircWave } \\
\text { P }\end{array}$} \\
\hline & d.f. & $F$ & $P$ & \\
\hline \multicolumn{5}{|l|}{ LD } \\
\hline untreated & 5,60 & 4.0806 & 0.0011 & 0.000185 \\
\hline DsRed $2^{\text {RNAi }}$ & 5,45 & 3.0001 & 0.0202 & 0.007221 \\
\hline$C W O^{R N A i}$ & 5,17 & 2.6963 & 0.057 & $>0.05$ \\
\hline \multicolumn{5}{|l|}{ DD } \\
\hline untreated & 5,14 & 5.9411 & 0.0038 & 0.001358 \\
\hline DsRed $2^{\text {RNAi }}$ & 5,26 & 10.7877 & 0.00001 & 0.0000 \\
\hline$C W O^{\text {RNAi }}$ & 5,23 & 1.7097 & 0.1724 & $>0.05$ \\
\hline \multicolumn{5}{|l|}{ LD } \\
\hline$t_{i m}^{\text {RNAi }}$ & 5,18 & 1.3930 & 0.2736 & $>0.05$ \\
\hline$p e r^{R N A i}$ & 5,12 & 0.5117 & 0.7626 & $>0.05$ \\
\hline$C / k^{\text {RNAi }}$ & 5,18 & 5.8265 & 0.0023 & $>0.05$ \\
\hline$c y c^{\mathrm{RNAi}}$ & 5,17 & 1.5834 & 0.2179 & $>0.05$ \\
\hline$C r y 2^{\text {RNAi }}$ & 5,27 & 3.0132 & 0.0274 & 0.010773 \\
\hline
\end{tabular}

expression profile of the clock genes per, tim, cry2, Clk, cyc, vri, Pdp1, E75, and HR3. We measured the mRNA levels of the genes in the optic lobe of adult male crickets, which were injected with dscwo in the abdomen and kept under LD or DD. The results are shown in Figs. 5 and 6 and Table 4. Under LD, the mRNA levels of per, tim, cyc, and cry 2 in control crickets treated with DsRed2 $2^{\mathrm{RNAi}}$ showed the expression profiles that were previously reported for untreated crickets: per, tim and cry 2 were rhythmically expressed with a peak during the night, while the levels of $c y c$ peaked during the day (Fig. 5, Table 4) $[16,18,22]$. The expression of $C l k$ was quasi-rhythmic (Fig. 5, Table 4), although it was previously reported to be constitutively expressed [23]. vri, Pdp1, and HR3 were also rhythmically expressed, while E75 was expressed quasirhythmically with a peak during the night (Fig. 5, Table 4). $c w o^{\mathrm{RNAi}}$ treatment significantly downregulated the expression of per, Clk, and $c y c$ to eliminate their daily rhythms (Fig. 5, Table 4). However, tim and cry2 maintained a clear rhythm of expression, but the rising phase was slightly delayed in tim, and the peak was slightly delayed in cry 2 (Fig. 5, Table 4). vri, Pdp1, E75, and HR3 were upregulated upon $c w o^{\mathrm{RNAi}}$ treatment. Their transcript levels stayed at levels similar to or higher than their peak levels in DsRed2 ${ }^{\mathrm{RNAi}}$-treated control crickets (Fig. 5).

Under DD, the mRNA expression profiles of the clock genes in the DsRed2 ${ }^{\mathrm{RNAi}}$ control crickets were basically similar to those observed under LD, except for Pdp1 and HR3; the former showed a rhythmic expression that peaked at mid-subjective night, while the latter was expressed essentially constitutively (Fig. 6, Table 4). The effects of $c w o^{\mathrm{RNAi}}$ were similar to those obtained under LD; however, tim, E75, and HR3 showed features different from those observed under LD (Fig. 6). Specifically, tim lost its oscillation, while E75 was significantly downregulated, and the levels of HR3 were similar to those observed in DsRed $2^{\mathrm{RNAi}}$-treated controls, implying that light plays a certain role in the clock oscillatory mechanism. A fluctuation was observed in the mRNA levels of $v r i$ and $P d p 1$, but the periodicity of the changes was not significant (Fig. 6, Table 4). Interestingly, cry2 retained a weak but significant oscillatory expression with a peak in late subjective night, similar to that under LD (Fig. 6, Table 4), suggesting its role in the generation of locomotor rhythm in $c w o^{\mathrm{RNAi}}$ crickets.

\section{Discussion}

\section{The cwo gene}

In the present study, we obtained the partial sequence of Gb'cwo from the RNA-seq data and verified its existence by cDNA cloning. Analysis of the sequence revealed that cwo of crickets is a member of the bHLH-ORANGE family. Expression analysis of cwo with qRT-PCR revealed that it is rhythmically expressed in the compound eye, optic lobe, and brain, suggesting that it is involved in rhythm generation in these tissues (Fig. 2). This result is consistent with our previous findings that the optic lobe is the locus of the circadian clock that controls locomotor rhythms [24], that the compound eye shows circadian rhythms in its sensitivity to light [25], and that the brain shows rhythmic expression of per and tim [26]. It is also expressed at some level in the subesophageal ganglion, but rather constitutively, suggesting that cwo may play a role other than its role in the circadian clock, similar to per and tim in the Drosophila gonads [27], and vri in larval molting and metamorphosis in the moth Helicoverpa armigera [28]. Further studies may reveal additional, non-clock functions of $c w o$.

\section{Regulation of cwo expression}

Our results showed that cwo is rhythmically expressed in the optic lobe, the clock tissue of crickets, with a peak in the middle of the night (Fig. 3). This result is consistent with reports from Drosophila $[6-8,12]$ suggesting that cwo is under the control of the circadian clock. A similar expression profile has been reported in the monarch butterfly $D$. plexippus [14], while no significant daily $c w o$ rhythm has been detected in the wasp $N$. vitripennis [15]. $c y c^{\mathrm{RNAi}}$ treatment led to strong downregulation of the expression of $c w o$ to the basal level of the DsRed $2^{\mathrm{R}-}$ ${ }^{N A i}$ control (Fig. 3c). Considering that cwo is transactivated by CLK/CYC through the E-box in Drosophila and that there are many E-box elements in the UTR regions of Gb'cwo (Fig. 1c), this result suggests that cricket cwo 

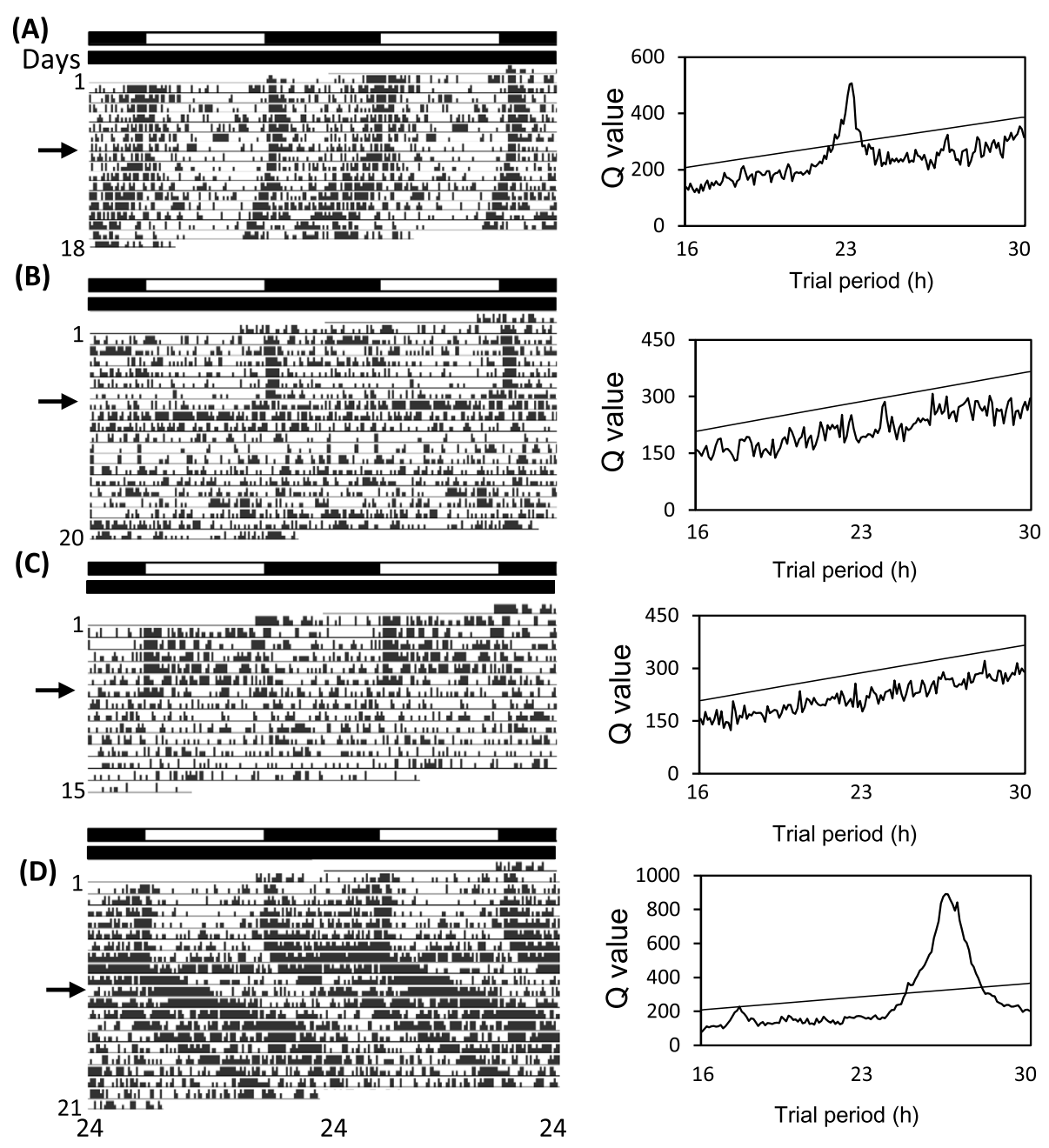

Time of day $(\mathrm{h})$

Fig. 4 Effects of dscwo on the locomotor rhythm in the cricket Gryllus bimaculatus under a light-dark cycle (LD) and in subsequent constant darkness (DD). The left and right panels show the double-plotted actograms and results of chi-square periodogram analysis, respectively. a: A representative control cricket treated with dsDsRed2, showing a clear nocturnal rhythm in LD and a free-running period slightly shorter than $24 \mathrm{~h}$ in the ensuing DD. $\mathbf{b}-\mathbf{d}$ : Locomotor rhythms of crickets injected with dscwo in the abdomen (b) or compound eyes (c, d). Most treated crickets showed a nocturnal rhythm (b, d), but some showed diurnal rhythms (c), in LD. Some of the crickets became arrhythmic on transfer to DD (b, $\mathbf{c})$, while the remaining crickets showed a free-running rhythm with a period longer than $24 \mathrm{~h}(\mathbf{d})$. Arrows indicate the day of transfer from $L D$ to DD. White and black bars above the actograms indicate light (white) and dark (black) fractions, respectively. See text for details

is transactivated by a similar mechanism, although we could not exclude the possibility that CYC affects $c w o$ expression via non-E-box mediated mechanisms. $C l k^{\mathrm{RNAi}}$ treatment did not eliminate the $c w o$ rhythm but shifted it by $8 \mathrm{~h}$, such that it peaked in the early morning without any reduction in transcript levels (Fig. 3c), whereas in Clk-knockout monarch butterflies, cwo was expressed at constitutively low levels [14]. This may be explained by the gradual accumulation of $C l k$ mRNA that survived RNAi treatment, with the resultant CLK/CYC complexes stimulating cwo transcription in a delayed time course, or by transactivation of $c w o$ by $\mathrm{CYC}$ alone in a delayed manner. Treatment with $C l k^{\mathrm{RNAi}}$ leads to arrhythmic locomotor activity and terminates the oscillation of the transcript levels of per and tim [23]. Therefore, as cwo oscillation survived the $C l k^{\mathrm{RNAi}}$ treatment, it is possible that cwo has no significant role in rhythm generation. These possibilities should be examined in future studies.

RNAi of per or tim was found to eliminate the daily rhythmic expression of $c w o$ (Fig. 3c). This effect may be caused indirectly through complex clock machinery. Since their RNAi downregulates the expression of $\mathrm{Clk}$ and $c y c[16,23]$, the decrease in the levels of CLK and CYC may in turn result in downregulation of the expression of $c w o$. per ${ }^{\mathrm{RNAi}}$ induces arrhythmicity in locomotor 


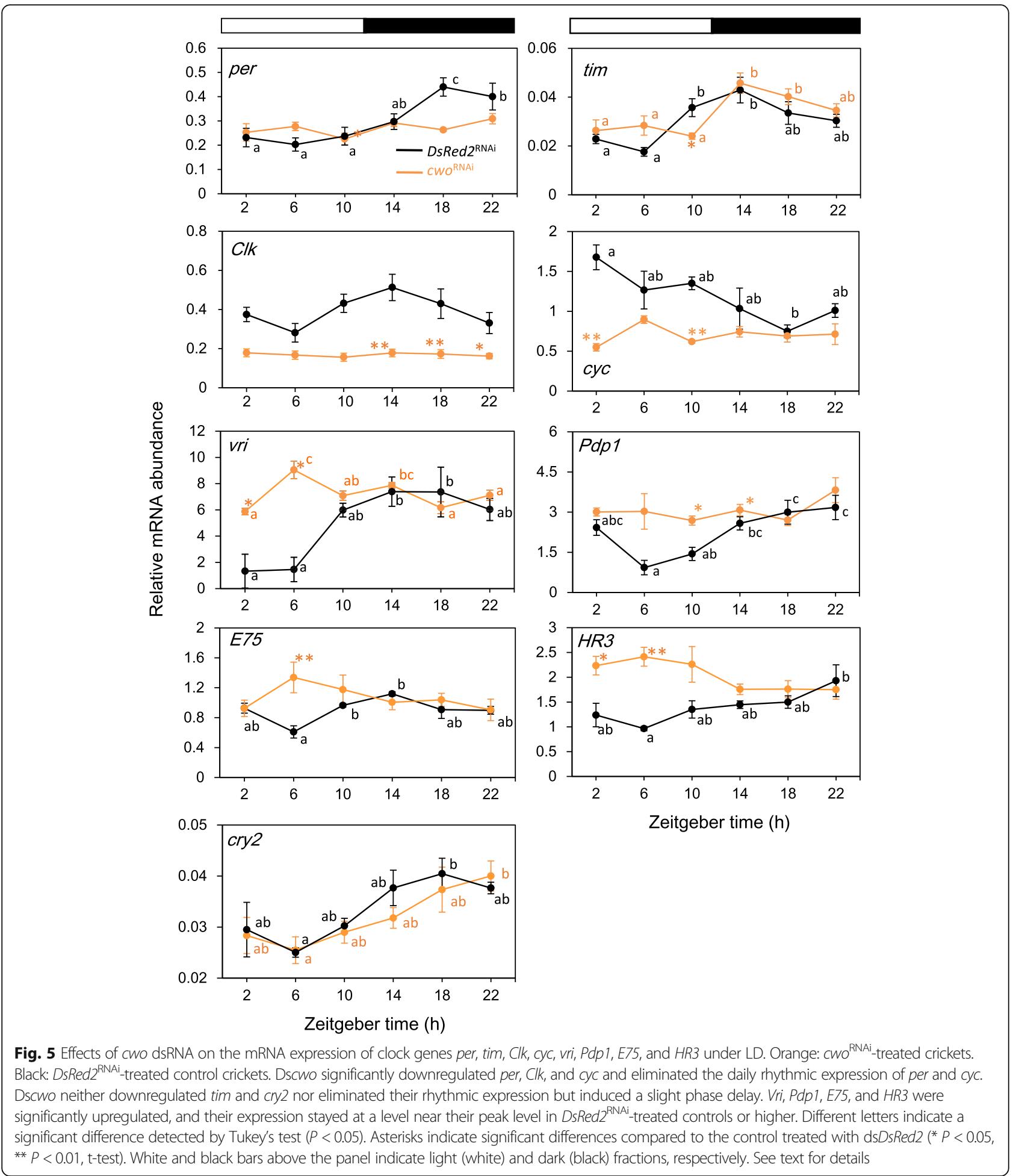

activity, while tim $^{\mathrm{RNAi}}$ shortens the free-running period of locomotor rhythms $[18,22]$. The maintenance of locomotor rhythm in tim $^{\mathrm{RNAi}}$ crickets is most likely attributable to oscillation of cry2 [17]. Thus, cwo oscillation may not be required for the cry 2 oscillation. This hypothesis is also supported by the results of this study, which show that cry 2 maintained its rhythmic expression in $c w o^{\mathrm{RNAi}}$ crickets under both LD and DD (Figs. 5 and 6). The present study also revealed that $\operatorname{cry} 2^{\mathrm{RNAi}}$ treatment had almost no effect on the rhythmic expression of $c w o$ (Fig. 3c), suggesting that cry 2 oscillation is independent of the main per/tim loop, including cwo (Fig. 7). 


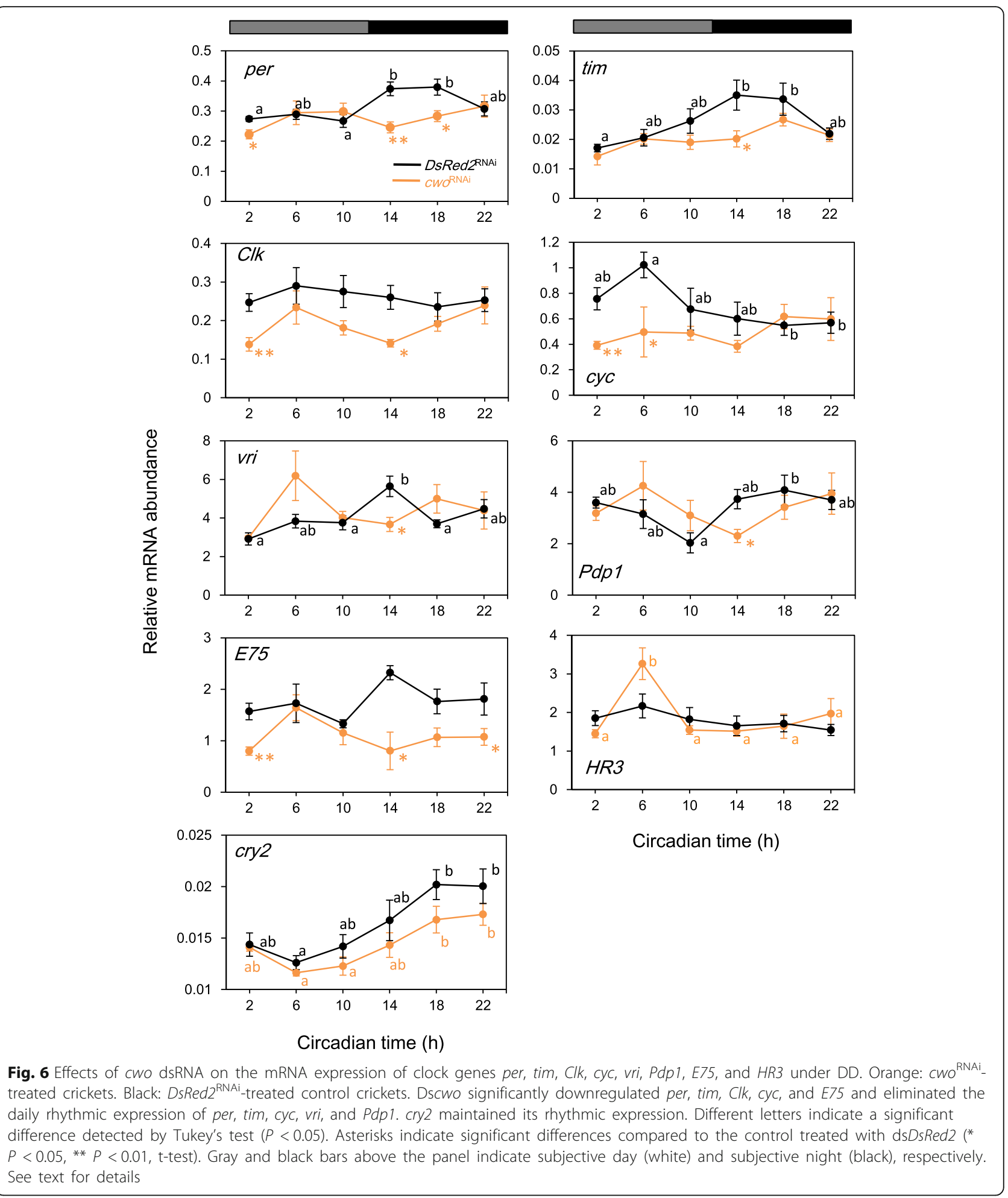

\section{Role of cwo in the clock machinery}

The role of $c$ wo has been extensively studied in Drosophila [6-8]. It is a transcription factor belonging to the bHLHORANGE family. The lack of $c w o$ results in a longer freerunning period of locomotor rhythm and a reduced amplitude of per and tim cycling [12]. It has been shown that CWO binds to the E-box during late night to midday in competition with CLK/CYC to inhibit the transcription of E-box-dependent genes, such as per and tim, increasing the amplitude of their daily expression rhythms [9]. 
Table 4 Results of statistical analyses of daily clock gene expression in crickets treated with DsRed2 ${ }^{\mathrm{RNAi}}$ (control) or $\mathrm{CwO}^{\mathrm{RNAi}}$ under light-dark cycle (LD) or constant darkness (DD)

\begin{tabular}{llllll}
\hline Clock & Treatment & \multicolumn{3}{l}{ ANOVA } & \\
\cline { 3 - 4 } genes & & d.f. & F & CircWave & P \\
\hline
\end{tabular}

LD

\begin{tabular}{|c|c|c|c|c|c|}
\hline \multirow[t]{2}{*}{ per } & DsRed $2^{\mathrm{RNAi}}$ & 5,44 & 6.4931 & 0.0001 & 0.00001 \\
\hline & $C W O^{\text {RNAi }}$ & 5,18 & 1.8144 & 0.1607 & $>0.05$ \\
\hline \multirow[t]{2}{*}{ tim } & DsRed $2^{\mathrm{RNAi}}$ & 5,16 & 5.8537 & 0.0029 & 0.000896 \\
\hline & $C W O^{\mathrm{RNAi}}$ & 5,17 & 6.2874 & 0.0018 & 0.001774 \\
\hline \multirow[t]{2}{*}{ Clk } & DsRed $2^{\text {RNAi }}$ & 5,15 & 2.298 & 0.0972 & 0.047724 \\
\hline & $C W O^{\text {RNAi }}$ & 5,17 & 0.2012 & 0.9575 & $>0.05$ \\
\hline \multirow[t]{2}{*}{$c y c$} & DsRed $2^{\mathrm{RNAi}}$ & 5,15 & 4.0251 & 0.0162 & 0.008703 \\
\hline & $C W O^{\text {RNAi }}$ & 5,17 & 2.6741 & 0.0584 & $>0.05$ \\
\hline \multirow[t]{2}{*}{ vri } & $\operatorname{DsRed} 2^{\mathrm{RNAi}}$ & 5,16 & 5.312 & 0.0046 & 0.00026 \\
\hline & $C W O^{\text {RNAi }}$ & 5,18 & 7.5919 & 0.0005 & $>0.05$ \\
\hline \multirow[t]{2}{*}{ Pdp1 } & $\operatorname{DsRed} 2^{\mathrm{RNAi}}$ & 5,48 & 6.9259 & 0.0001 & 0.000004 \\
\hline & $C W O^{\text {RNAi }}$ & 5,18 & 1.2890 & 0.3119 & $>0.05$ \\
\hline \multirow[t]{2}{*}{ E75 } & DsRed $2^{\text {RNAi }}$ & 5,16 & 4.2222 & 0.0122 & $>0.05$ \\
\hline & $C W O^{\text {RNAi }}$ & 5,17 & 1.3585 & 0.2883 & $>0.05$ \\
\hline \multirow[t]{2}{*}{ HR3 } & DsRed $2^{\text {RNAi }}$ & 5,18 & 2.8840 & 0.0439 & 0.031795 \\
\hline & $C W O^{\text {RNAi }}$ & 5,17 & 2.4046 & 0.0801 & 0.007331 \\
\hline \multirow[t]{2}{*}{ cry2 } & DsRed $2^{\text {RNAi }}$ & 5,15 & 4.2396 & 0.0133 & 0.000402 \\
\hline & $c W O^{\text {RNAi }}$ & 5,17 & 3.2741 & 0.0298 & 0.00397 \\
\hline \multicolumn{6}{|c|}{ DD } \\
\hline \multirow[t]{2}{*}{ per } & $\operatorname{DsRed} 2^{\mathrm{RNAi}}$ & 5,20 & 5.6718 & 0.0020 & 0.000773 \\
\hline & $C W O^{\text {RNAi }}$ & 5,16 & 1.6874 & 0.1947 & $>0.05$ \\
\hline \multirow[t]{2}{*}{ tim } & DsRed $2^{\mathrm{RNAi}}$ & 5,26 & 3.8506 & 0.0096 & 0.000482 \\
\hline & $C W O^{\text {RNAi }}$ & 5,14 & 2.4257 & 0.0878 & $>0.05$ \\
\hline \multirow[t]{2}{*}{ Clk } & $\operatorname{DsRed} 2^{\mathrm{RNAi}}$ & 5,27 & 0.3144 & 0.8999 & $>0.05$ \\
\hline & $C W O^{\mathrm{RNAi}}$ & 5,17 & 2.3294 & 0.0885 & $>0.05$ \\
\hline \multirow[t]{2}{*}{$c y c$} & DsRed $2^{\text {RNAi }}$ & 5,28 & 2.9222 & 0.0303 & 0.007461 \\
\hline & $C W O^{\text {RNAi }}$ & 5,16 & 1.0490 & 0.4235 & $>0.05$ \\
\hline \multirow[t]{2}{*}{$v r i$} & DsRed $2^{\text {RNAi }}$ & 5,25 & 5.3741 & 0.0017 & 0.023523 \\
\hline & $C W O^{\text {RNAi }}$ & 5,13 & 2.1364 & 0.1254 & $>0.05$ \\
\hline \multirow[t]{2}{*}{ Pdpl } & $\operatorname{DsRed} 2^{\mathrm{RNAi}}$ & 5,23 & 2.9852 & 0.0321 & 0.020129 \\
\hline & $C W O^{\text {RNAi }}$ & 5,17 & 1.2542 & 0.3280 & $>0.05$ \\
\hline \multirow[t]{2}{*}{ E75 } & DsRed $2^{\text {RNAi }}$ & 5,24 & 2.2516 & 0.0818 & $>0.05$ \\
\hline & $C W O^{R N A i}$ & 5,18 & 1.6580 & 0.1957 & $>0.05$ \\
\hline \multirow[t]{2}{*}{ HR3 } & DsRed $2^{\text {RNAi }}$ & 5,27 & 0.7698 & 0.5798 & $>0.05$ \\
\hline & $C W O^{\text {RNAi }}$ & 5,17 & 5.7916 & 0.0027 & $>0.05$ \\
\hline \multirow[t]{2}{*}{ cry2 } & DsRed $2^{\text {RNAi }}$ & 5,30 & 5.1756 & 0.0015 & 0 \\
\hline & $C W O^{\text {RNAi }}$ & 5,17 & 5.3303 & 0.0040 & 0.000107 \\
\hline
\end{tabular}

The results of this study showed that the locomotor rhythm phenotypes of $c w o{ }^{\mathrm{RNAi}}$ crickets are quite similar to those reported for cwo-deficient Drosophila mutant flies. They showed either arrhythmic activity or rhythms with longer free-running periods (Fig. 4), suggesting that cwo plays an important role in the cricket clock mechanism. The effects of $c w o^{\mathrm{RNAi}}$ at the molecular level were more severe than those found in cwo-deficient flies: under $\mathrm{DD}, c w o^{\mathrm{RNAi}}$ downregulated most E-boxregulated clock genes, including per, tim, and E75, and eliminated the expression rhythm in per, tim, vri, Pdp1, and $E 75$ (Fig. 6). These effects may be explained by the regulation of gene transcription by CWO through the Ebox-dependent transcription factors CLK and CYC, generating robust rhythmic expression, as in Drosophila [12] (Fig. 7). In fact, $c w o^{\mathrm{RNAi}}$ significantly downregulated the expression of $C l k$ and $c y c$ and eliminated the rhythmic expression of $c y c$ (Fig. 6). Therefore, cwo may function as a transcriptional activator (Fig. 7), as has been suggested in Drosophila [6-8, 12], although the mechanism of transcriptional activation by CWO is currently unknown. CWO may activate transcription of the E-box mediated clock genes by enhancing the transcriptional activity of CLK and CYC (Fig. 7). Alternatively, it may activate the transcription of $\mathrm{Clk}$ and $c y c$, which in turn activate the E-box mediated clock genes.

Our data were obtained via RNAi-mediated gene silencing experiments and were not fully compatible with the results obtained by genetic manipulation in Drosophila. Nonetheless, the regulatory role of CWO in each of the clock-relevant genes may be different between crickets and Drosophila. Further studies are required to resolve this issue.

Importantly, cry 2 maintained its rhythmic expression upon $c w o^{\mathrm{RNAi}}$ treatment, even after the rhythmic expression of all other clock genes was interrupted (Figs. 5 and 6). The maintenance of locomotor rhythms in $c w o^{\mathrm{RNAi}}$ crickets is most likely attributable to the $c r y 2$ rhythm. This finding is consistent with our previously proposed hypothesis that cry2 forms a transcriptional/translational feedback loop that can function independently of the per/tim oscillatory loop in the cricket [17] (Fig. 7).

Interestingly, $c w o^{\mathrm{RNAi}}$ treatment revealed that light modulates the oscillatory system. The treatment reduced the transcript levels of $E 75$ and had no significant effect on the transcript level of HR3 under DD, while those levels were significantly higher than the control under LD (Figs. 5 and 6). These observations suggest that light somehow modulates the transcription of these genes. In addition, tim was rhythmically expressed under LD at a level similar to that in control crickets treated with $\operatorname{DsRed2^{\mathrm {RNAi}}}$ but with the rising phase slightly delayed (Fig. 5). tim is known to maintain its rhythmic expression even when other clock genes are arrhythmically expressed at low levels due to double RNAi of cry 1 and cry2 [17]. Thus, the mechanism regulating tim cycling may differ from those for other E-box mediated clock 


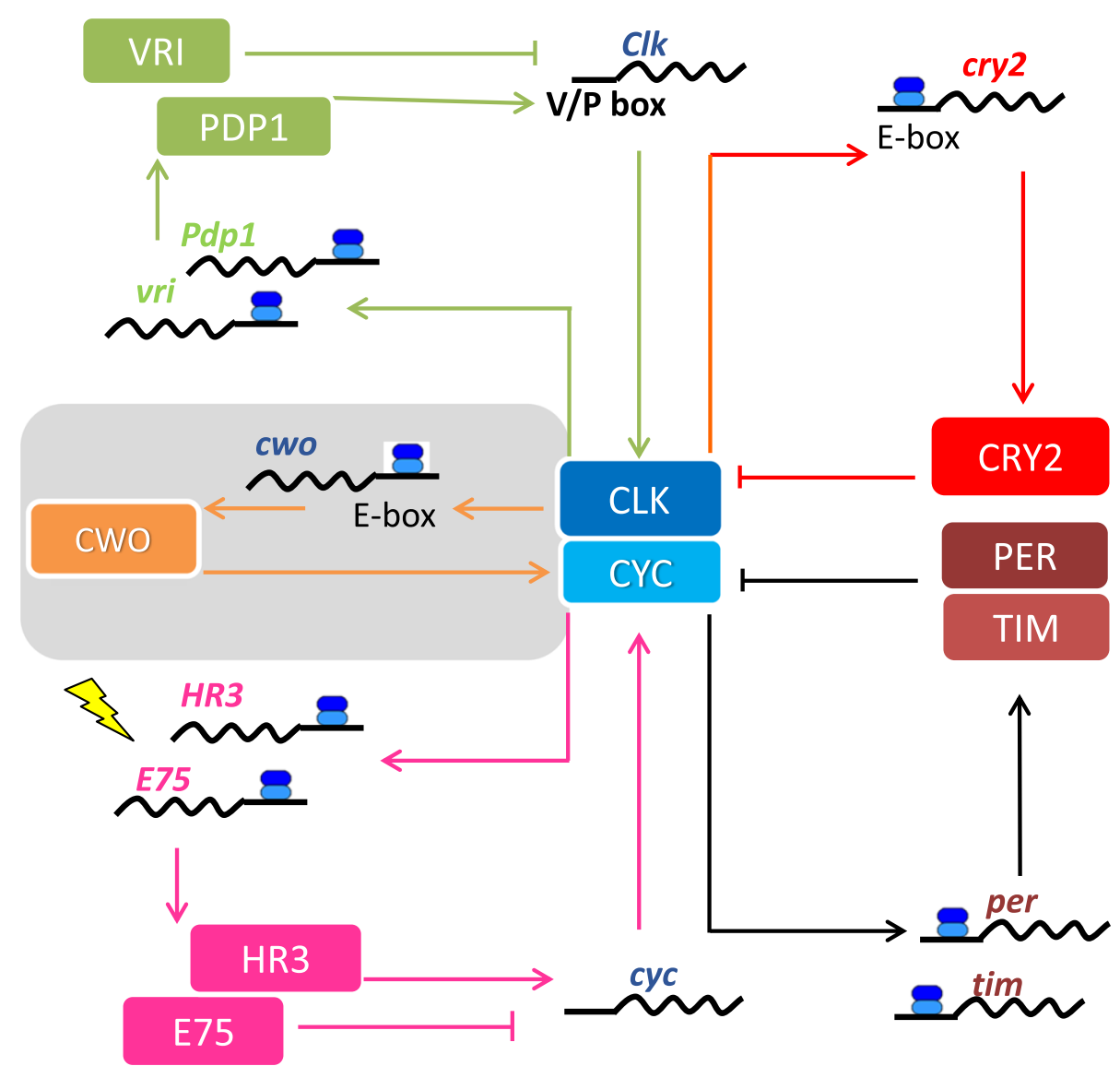

Fig. 7 A model of the clock oscillatory mechanism in the cricket Gryllus bimaculatus. The clock most likely oscillates based on transcriptional/ translational feedback loops. Transcription of per, tim, cry2, vri, Pdp 1, E75, and HR3 is activated by CLK and CYC through the E-box. The protein products PER and TIM form heterodimers and suppress the transcriptional activity of CLK/CYC, while CRY2 also suppresses CLK/CYC through a pathway that is different from that of PER/TIM. Previous results have suggested that both Clk and cyc could be rhythmically expressed. According to the circadian oscillatory mechanism of Drosophila and firebrats [4, 33], VRI and E75 suppress, while PDP1 and HR3 activate, the transcription of Clk and cyc, respectively. CWO probably enhances the transcription of CLK/CYC target genes through upregulation of Clk and cyc. When cwo is downregulated, light promotes the expression of HR3 and E75. See text for details

genes. In cwo-deficient Drosophila mutants, the falling phase of tim is reported to be slightly delayed [6], suggesting that the mechanism of tim regulation by cwo differs between the two species. Although the mechanisms underlying these light-dependent changes in clock gene expression are currently unclear, they contribute to the maintenance of a molecular rhythm in $c w o^{\mathrm{RNAi}}$ crickets to generate robust daily behavioral rhythms under LD, together with the cry2 loop, which persists under both LD and DD. While further study is necessary to clarify the underlying mechanism, our results are reminiscent of the light-dependent induction of tim expression in Drosophila [29].

Apart from its function in the oscillatory mechanism, the mammalian cwo homolog Dec1 is known to play an essential role in the phase resetting mechanism of peripheral clocks [30]. In this mechanism, Dec1 is induced through TGF/ALK5/SMAD signaling but independent of Per1/Per2 induction, which is essential for lightevoked phase resetting [31]. Although we currently have no direct evidence for the involvement of cwo in clock phase resetting in cricket, this issue should be addressed in future studies since the cricket clock is known to be reset by a nonphotic but rather temperature-dependent mechanism [32].

\section{Conclusions}

In this study, we have shown that cwo in the cricket Gryllus bimaculatus is a clock gene belonging to the bHLH-ORANGE family and is rhythmically expressed in the clock tissue, the optic lobes, and peaks during the night under the LD cycle. Cwo plays an important role in the regulation of behavioral rhythms, as $c w o^{\mathrm{RNAi}}$ resulted in arrhythmicity or elongation of the free-running period of locomotor rhythms. This alteration in behavioral rhythms is most likely caused by changes in the 
molecular oscillatory mechanism; in $c w o^{\mathrm{RNAi}}$ crickets, the expression of most clock genes became arrhythmic, and $c r y 2$ alone retained rhythmic expression under DD. Based on these results, we propose that cwo is a component of the per/tim oscillatory loop (Fig. 7). To our knowledge, this is the first study on the function of $c w o$ in insects other than Drosophila. There are some differences in the role of cwo between Drosophila and crickets. Since crickets are hemimetabolous and are phylogenetically more basal than Drosophila, the role of cricket cwo may be more ancestral than that in Drosophila. To understand the general role of cwo in insect clocks, its functions should be compared among different groups of insects.

\section{Acknowledgments}

We thank Dr. Akira Matsumoto of Juntendo University for critical reading of the manuscript. We also thank the members of the Chronobiology Laboratory for their discussion on this research project.

\section{Authors' contributions}

YT and KT designed the experiments. YT, TS, and MM performed the experiments. TB and TM performed the analysis of the G'cwo gene. YT and $\mathrm{KT}$ analyzed the data and wrote the manuscript. The author(s) read and approved the final manuscript.

\section{Funding}

This study was supported by the Japan Society for Promotion of Science (JSPS) Grants-in-Aid for Scientific Research No. JP15H04400 and JP18H02480 to KT.

\section{Availability of data and materials}

The datasets supporting the conclusions of this article are included within the article.

\section{Ethics approval and consent to participate}

Not applicable.

\section{Consent for publication}

Not applicable.

\section{Competing interests}

The authors declare that they have no competing interests.

\section{Author details}

'Graduate School of Natural Science and Technology, Okayama University, Okayama 700-8530, Japan. ${ }^{2}$ Graduate School of Medicine, Dentistry and Pharmaceutical Science, Okayama University, Okayama 700-8558, Japan. ${ }^{3}$ Graduate School of Technology, Industrial and Social Sciences, Tokushima University, Tokushima 770-8513, Japan.

Received: 15 June 2020 Accepted: 31 October 2020 Published online: 11 November 2020

\section{References}

1. Tomioka K, Matsumoto A. Chapter three - the circadian system in insects: cellular, molecular, and functional organization. Adv Insect Physiol. 2019;56: 73-115.

2. Tomioka K, Matsumoto A. Circadian molecular clockworks in non-model insects. Curr Opin Insect Sci. 2015;7(February):58-64.

3. Blau J, Young MW. Cycling vrille expression is required for a functional Drosophila clock. Cell. 1999;99(6):661-71.

4. Cyran SA, Buchsbaum AM, Reddy KL, Lin M-C, Glossop NRJ, Hardin PE, Young MW, Storti RV, Blau J. vrille, Pdp1 and dClock form a second feedback loop in the Drosophila circadian clock. Cell. 2003;112(3):329-41.
5. Glossop NR, Houl JH, Zheng H, Ng FS, Dudek SM, Hardin PE. VRILLE feeds back to control circadian transcription of Clock in the Drosophila circadian oscillator. Neuron. 2003;37(2):249-61.

6. Kadener S, Stoleru D, McDonald M, Nawathean P, Rosbash M. Clockwork Orange is a transcriptional repressor and a new Drosophila circadian pacemaker component. Genes Dev. 2007;21(13):1675-86.

7. Lim C, Chung BY, Pitman JL, McGill JJ, Pradhan S, Lee J, Keegan KP, Choe J, Allada R. clockwork orange encodes a transcriptional repressor important for circadian-clock amplitude in Drosophila. Curr Biol. 2007;17(12):1082-9.

8. Matsumoto A, Ukai-Tadenuma M, Yamada RG, Houl J, Uno KD, Kasukawa T, Dauwalder B, Itoh TQ, Takahashi K, Ueda R, et al. A functional genomics strategy reveals clockwork orange as a transcriptional regulator in the Drosophila circadian clock. Genes Dev. 2007;21(13):1687-700.

9. Zhou J, Yu W, Hardin PE. CLOCKWORK ORANGE enhances PERIOD mediated rhythms in transcriptional repression by antagonizing E-box binding by CLOCK-CYCLE. PLoS Genet. 2016;12(11):e1006430.

10. Honma S, Kawamoto T, Takagi Y, Fujimoto K, Sato F, Noshiro M, Kato Y. Honma K-i: Dec1 and Dec2 are regulators of the mammalian molecular clock. Nature. 2002;419(6909):841-4.

11. Sato F, Kawamoto T, Fujimoto K, Noshiro M, Honda KK, Honma S. Honma Ki, Kato Y. Functional analysis of the basic helix-loop-helix transcription factor DEC1 in circadian regulation. Eur J Biochem. 2004;271(22):4409-19.

12. Richier B, Michard-Vanhée C, Lamouroux A, Papin C, Rouyer F. The clockwork orange Drosophila protein functions as both an activator and a repressor of clock gene expression. J Biol Rhythm. 2008;23(2):103-16.

13. Ingram KK, Kutowoi A, Wurm Y, Shoemaker D, Meier R, Bloch G. The molecular clockwork of the fire ant Solenopsis invicta. PLoS One. 2012;7(11): e45715.

14. Lugena AB, Zhang Y, Menet JS, Merlin C. Genome-wide discovery of the daily transcriptome, DNA regulatory elements and transcription factor occupancy in the monarch butterfly brain. PLoS Genet. 2019;15(7):e1008265.

15. Mukai A, Goto SG. The clock gene period is essential for the photoperiodic response in the jewel wasp Nasonia vitripennis (Hymenoptera: Pteromalidae). Appl Entomol Zool. 2016;51(2):185-94.

16. Uryu O, Karpova SG, Tomioka K. The clock gene cycle plays an important role in the circadian clock of the cricket Gryllus bimaculatus. J Insect Physiol. 2013;59(7):697-704.

17. Tokuoka A, Itoh TQ, Hori S, Uryu O, Danbara Y, Nose M, Bando T, Tanimura T. Tomioka K: cryptochrome genes form an oscillatory loop independent of the per/tim loop in the circadian clockwork of the cricket Gryllus. bimaculatus. Zool Lett. 2017;3(April 6):5.

18. Moriyama Y, Sakamoto T, Karpova SG, Matsumoto A, Noji S, Tomioka K. RNA interference of the clock gene period disrupts circadian rhythms in the cricket Gryllus bimaculatus. J Biol Rhythm. 2008;23(4):308-18.

19. Sokolove $P G$, Bushell WN. The chi square periodogram: its utility for analysis of circadian rhythm. J Theor Biol. 1978;72(1):131-60.

20. Schmid B, Helfrich-Förster C, Yoshii T. A new ImageJ plug-in "ActogramJ" for chronobiological analyses. J Biol Rhythm. 2011;26(5):464-7.

21. Kaneko M, Park JH, Cheng Y, Hardin PE, Hall JC. Disruption of synaptic transmission or clock-gene-product oscillations in circadian pacemaker cells of Drosophila cause abnormal behavioral rhythms. J Neurobiol. 2000;43(3): 207-33.

22. Danbara Y, Sakamoto T, Uryu O, Tomioka K. RNA interference of timeless gene does not disrupt circadian locomotor rhythms in the cricket Gryllus bimaculatus. J Insect Physiol. 2010;56:1738-45.

23. Moriyama Y, Kamae Y, Uryu O, Tomioka K. Gb'Clock is expressed in the optic lobe and required for the circadian clock in the cricket Gryllus bimaculatus. J Biol Rhythm. 2012;27:467-77.

24. Tomioka K, Chiba Y. Effects of nymphal stage optic nerve severance or optic lobe removal on the circadian locomotor rhythm of the cricket, Gryllus bimaculatus. Zool Sci. 1984;1(3):375-82.

25. Tomioka K, Chiba Y. Persistence of circadian ERG rhythms in the cricket with optic tract severed. Naturwissenschaften. 1982;69(8):355-6.

26. Uryu O, Tomioka K. Circadian oscillations outside the optic lobe in the cricket Gryllus bimaculatus. J Insect Physiol. 2010;56(9):1284-90.

27. Beaver LM, Rush BL, Gvakharia BO, Giebultowicz JM. Noncircadian regulation and function of clock genes period and timeless in oogenesis of Drosophila melanogaster. J Biol Rhythm. 2003;18:463-72.

28. Liu X, Zhang S, Shen ZJ, Liu Y, Li Z, Liu X. Vrille is required for larval moulting and metamorphosis of Helicoverpa armigera (Lepidoptera: Noctuidae). Insect Mol Biol. 2019;28(3):355-71. 
29. Chen W-F, Majercak J, Edery I. Clock-gated photic stimulation of timeless expression at cold temperatures and seasonal adaptation in Drosophila. J Biol Rhythm. 2006;21(4):256-71.

30. Kon N, Hirota T, Kawamoto T, Kato Y, Tsubota T, Fukada Y. Activation of TGF- $\beta$ /activin signalling resets the circadian clock through rapid induction of Dec1 transcripts. Nat Cell Biol. 2008;10(12):1463-9.

31. Schwartz WJ, Tavakoli-Nezhad M, Lambert CM, Weaver DR, de la Iglesia HO. Distinct patterns of Period gene expression in the suprachiasmatic nucleus underlie circadian clock photoentrainment by advances or delays. Proc Natl Acad Sci U S A. 2011;108(41):17219-24.

32. Ikeda M, Tomioka K. Temperature dependency of the circadian locomotor rhythm in the cricket Gryllus bimaculatus. Zool Sci. 1993;10:597-604.

33. Kamae Y, Uryu O, Miki T, Tomioka K. The nuclear receptor genes HR3 and E75 are required for the circadian rhythm in a primitive insect. PLoS One. 2014;9(12):e114899.

\section{Publisher's Note}

Springer Nature remains neutral with regard to jurisdictional claims in published maps and institutional affiliations.

Ready to submit your research? Choose BMC and benefit from:

- fast, convenient online submission

- thorough peer review by experienced researchers in your field

- rapid publication on acceptance

- support for research data, including large and complex data types

- gold Open Access which fosters wider collaboration and increased citations

- maximum visibility for your research: over $100 \mathrm{M}$ website views per year

At BMC, research is always in progress.

Learn more biomedcentral.com/submissions 